\title{
In vivo bone tunnel remodeling in symptomatic patients after ACL reconstruction: a retrospective comparison of articular and extra-articular fixation
}

\author{
Dominic T. Mathis ${ }^{1}$ \\ Helmut Rasch ${ }^{2}$ \\ Michael T. Hirschmann 1 \\ 1 Department of Orthopaedic Surgery and Trauma- \\ tology, Kantonsspital Baselland, Bruderholz, \\ Switzerland \\ 2 Institute of Radiology and Nuclear Medicine, Kan- \\ tonsspital Baselland, Bruderholz, Switzerland
}

Corresponding author:

Dominic Thierry Mathis

Department of Orthopaedic Surgery and Traumatology, Kantonsspital Baselland (Bruderholz, Liestal, Laufen)

4101 Bruderholz, Switzerland

E-mail: dominic.mathis@unibas.ch

\section{Summary}

Background: there is only a paucity of studies dealing with bone remodeling within the tunnels after anterior cruciate ligament (ACL) reconstruction. The objective of this study was to evaluate the influence of tendon graft type and surgical fixation technique on bone tunnel remodeling in patients with symptomatic knees after $A C L$ reconstruction.

Methods: in a retrospective study $99 \mathrm{mTC}-\mathrm{HDP}$ bone tracer uptake (BTU) in SPECT/CT of 57 knees with symptoms of pain and/or instability after $A C L$ reconstruction was investigated. All $\mathbf{5 7}$ knees were subdivided according their anatomy (femur and tibia), fixation (articular versus extraarticular fixation) and graft types into eight groups: femoral-articular versus extra-articular fixation using bone-patellar tendon-bone (BPTB) and hamstring autografts; tibial-articular versus extra-articular fixation using patellar tendon and hamstring autografts; BTU grading for each area of the localisation scheme were recorded. Tunnel diameter and length was measured in the CT scans.

Results: BTU was higher for the articular fixation in the femur and for the extra-articular fixation in the tibial tunnel. Patellar tendon graft fixation showed a significantly higher BTU in the superior-lateral and posterior-central area of the tibia, meaning the areas of the tibial tunnel near the entrance into the joint. Tunnel enlargement correlated significantly with increased BTU $(p<0.05)$.

Conclusion: assessment of in vivo bone tunnel remodelling in symptomatic patients after ACL reconstruction revealed different patterns of BTU with regards to graft and fixation method.

KEY WORDS: ACL reconstruction, bone tunnel remodeling, fixation technique, hamstring graft, patellar tendon graft, SPECT/CT.

\section{Introduction}

Integration of the grafts and remodeling of the bone tunnels are essential parts of healing after reconstructive ligament surgery. However, there is only a paucity of studies dealing with bone tunnel remodeling after $A C L$ reconstruction.

Ishibashi et al. described histological changes in patellar tendon autografts, which occur over time within the tibial tunnel in patients undergoing revision ACL reconstruction ${ }^{1}$. They concluded that during remodeling the original bone-tendon junction of the ACL graft moves to the proximal tunnel wall. Those findings are in accordance with the results of Komiyama et al. who reported that bone graft cells migrate into the tendon graft and consequently contribute to the early stage remodeling of the tendon grafts in an animal model ${ }^{2}$. This finding is also in agreement with the results of Walsh et al., which showed in histological observations that tendon-bone healing stages of autologous tendon grafts were speed up in a rodent model after introducing gaps within the tendon graft prior to ACL reconstruction allowing host cells to infiltrate. In addition, the authors postulated that graft necrosis, usually observed in the initial two weeks of the healing process, was averted. However, these findings did not translate into improved biomechanical properties ${ }^{3}$. In contrast, a recent study was not able to prove the enhancement of the integration process in the rabbit model by transplanting bone marrow-derived mesenchymal stem cells alone ${ }^{4}$.

In 2014 Shearer et al. have tried to determine the geometrical arrangement of the fascicles in porcine ACL and patellar tendons using phosphotungstic acid (PTA) and iodine solution (IKI) as a method of enhancing contrast in the X-ray computed tomography ${ }^{5}$. They have shown that IKI appears to penetrate the 
In vivo bone tunnel remodeling in symptomatic patients after ACL reconstruction: a retrospective comparison of articular and extra-articular fixation

$\mathrm{ACL}$ and patellar tendon samples more effectively than PTA and postulated that in future this technique could be used to determine the collagen volume fraction and fascicle alignment in any tendon or ligament sample to predict mechanical behavior as a function of microstructure.

Bone tunnel enlargement is a commonly seen phenomenon after ACL reconstruction, but its exact causes are still under debate $e^{6,7}$. Most likely it is multifactorial due to both mechanical and biological causes. Another group demonstrated a significant association of femoral tunnel widening with a higher stiffness of the graft and hypertrophy of the graft throughout the remodeling process ${ }^{8}$. No correlation was found between tunnel widening and increased anteroposterior translation. Therefore, they postulated that tunnel enlargement is at least to some extent due to graft enlargement, which then results in a significant increase of bone density in the surrounding wall. However, this could also be due to the reaming of the tunnels leading to compression of bone around the tunnel wall.

A more recent $\mathrm{CT}$ study evaluated the progression of hydroxyapatite-poly-L-lactic acid (HA-PLLA) interference screw degradation and graft remodeling in patients after ACL reconstruction ${ }^{9}$. Nearly all screws were resorbed three to four years after ACL reconstruction with patellar tendon autograft or allograft. No tunnel widening, sclerosis or cysts were observed. In a landmark study Weiler et al. observed that during the early healing stages the weakest link for ACL fixation was the graft incorporation at the tunnel entrance. This led to a critical decrease in mechanical properties $^{10}$.

In a recent study it was shown that bone tracer uptake (BTU) intensity and distribution in 99mTc-HDPSPECT/CT correlated with the position and orientation of the tibial and femoral tunnels in patients after ACL reconstruction. However, correlation of instability, pivot shift and clinical laxity with 99mTc-HDPSPECT/CT tracer uptake intensity and distribution was not significant ${ }^{11}$. A previously published localisation scheme for anatomical localisation of the SPECT/CT BTU was used ${ }^{12}$. The causes of these results are not yet fully determined, but it seems most likely to be related to altered bone remodeling processes ${ }^{11}$.

In literature an active debate is focused on articular versus extra-articular tendon graft fixation as well as the graft choice itself ${ }^{13-17}$. It is still unclear which graft type and fixation methodis superior.

The primary purpose of this study was to investigate differences in bone tunnel remodeling of patients after $A C L$ reconstruction using hamstring or bone tendon bone autografts. The secondary purpose was to assess differences in bone tunnel remodeling between patients, in which the ACL had been fixed far from the joint (extra-articular) or close to the joint (articular). The hypothesis was that there is a significant difference in bone tunnel remodeling with regards to the fixation method, graft and tunnel diameter.

\section{Material and methods}

A consecutive series of patients $(n=57)$ with symptoms of pain and/or instability after ACL reconstruction were retrospectively included. Of the 57 patients, 40 were male and 17 were female (mean age \pm SD $27 \pm 11$ years, range $18-55$ years to 55 years age at surgery). The mean \pm SD time from surgery was $7 \pm 6$ years. Patients with previous intra-articular surgery of the knee joint were excluded. In view of the fact that Hirschmann et al. could not show any significances of BTU and clinical outcomes in their recent study the authors of this study have explicitly renounced assessing clinical outcome ${ }^{11}$. The study was approved by the local ethical committee.

In 35 knee sipsi lateral patellar tendon autograft sand in 22 knees hamstring autografts (semitendinosus and/or gracilis tendon) were used.

All 57 knees were subdivided according their anatomy (femur and tibia), fixation (articular versus extraarticular fixation) and graft types into eight groups:

- Femoral: $n=18$ articular fixation using hamstrings $(n=9)$ or patellar tendon $(n=9)$ autografts; $n=39$ extra-articular fixation using hamstrings $(n=13)$ or patellar tendon autograft $(n=26)$.

- Tibial: $n=24$ articular fixation using hamstrings $(n=10)$ and patellar tendon autograft $(n=14) ; n=32$ extra-articular fixation using hamstrings $(n=11)$ and patellar tendon autograft $(n=21)$.

Femoral fixation was achieved by a post screw $(n=20)$, an interference screw $(n=17)$, press-fit fixation $(n=4)$, endobutton $(n=10)$ and others $(n=6)$. Tibial fixation was achieved by a post screw $(n=27)$, an interference screw $(n=23)$, a tibial button $(n=3)$, a staple $(n=2)$ and others $(n=2)$. In both groups interference screw fixation was included in the articular fixation group, post screw, press-fit, endobutton, tibial button, staple fixation and others were subordinated to the group of extra-articular fixation. The decision of fixation method and graft type was taken by the patient and surgeon together. All ACL reconstructions were performed by experienced senior orthopaedic surgeons.

Demographic data of all patients were noted including gender, age, date of initial ACL reconstruction, time between surgery and SPECT/CT, type of ACL reconstruction, graft type and tibial and femoral fixation technique. Bone tunnel remodeling was assessed using BTU of SPECT/CT. 99mTc-HDP-SPECT/CT was performed in the delayed phase three to four hours after tracer injection (matrix size $128 \times 128$, angle step 32, and time per frame $25 \mathrm{~s}$ ). The BTU activity in SPECT/CT was analysed using a specialised software allowing 3D volumetric quantitative analysis of SPECT data as previously published ${ }^{18}$. A previously published localisation scheme (Fig. 1) for anatomical localisation of the SPECT/CT tracer activity was used ${ }^{12}$. Mean, standard deviation, minimum and maximum of maximum BTU grading for each area of the localisation scheme were recorded. For analysis BTU was normalised in relation to BTU in the mid shaft of the femur.

The diameter in different sections of the tibial and femoral tunnel was measured using CT images. 


\section{Statistical analysis}

Data were analysed using SPSS 17.0 (SPSS, Chicago, USA). In order to test influence of two factors in the same time a two factor variance analysis (ANOVA) was done in each case. Beneath descriptive statistics, Pearson's chi-square test of SPECT/CT BTU (intensity and distribution) with the fixation graft was performed $(p<0.05)$. Spearman's rho test and Pearson's correlation was used for lineal correlation analysis of SPECT/CT tracer uptake and tunnel enlargement. The study meets the ethical standards of the Journal ${ }^{19}$.

\section{Results}

99mTc-HDP-SPECT/CT BTU in the femoral and tibial tunnels with regards to graft and fixation type are presented in Table 1. Slightly higher BTU was found for the articular fixation in the femur and for the extra-ar- ticular fixation in the tibial tunnel. Type of fixation or type of used graft showed no significant correlation with 99mTc-HDP-BTU in SPECT/CT (Tab. 1, Fig. 2). No significant correlation of fixation and graft type with $99 \mathrm{mTC}-\mathrm{HDP}-\mathrm{BTU}$ in SPECT/CT was found for the femoral subgroup (Tabs. 2, 3).

Significant correlations of the standardised maximum intensity and distribution of $99 \mathrm{mTc}$-HDP-BTU in SPECT/CT was found for various anatomical knee regions (Tab. 4). Patellar tendon graft fixation induced a significantly higher tracer uptake in the superior-lateral (ap.tib.1s) and posterior-central (ax.tib.3p) tibial regions (Fig. 3), meaning the entrance site of the tunnel into the joint. Trends towards significance were shown in superior-central and lateral regions of the tibia (ap.tib.3s/axial.tib.1p,1a).

More significances $(p<0.05)$ and tendencies $(p<0.1)$ were revealed for the standardised mean intensity of the tibia: higher SPECT/CT uptake for extra-articular fixation and patellar-tendon graft than for the hamstring fixation (Tab. 5).

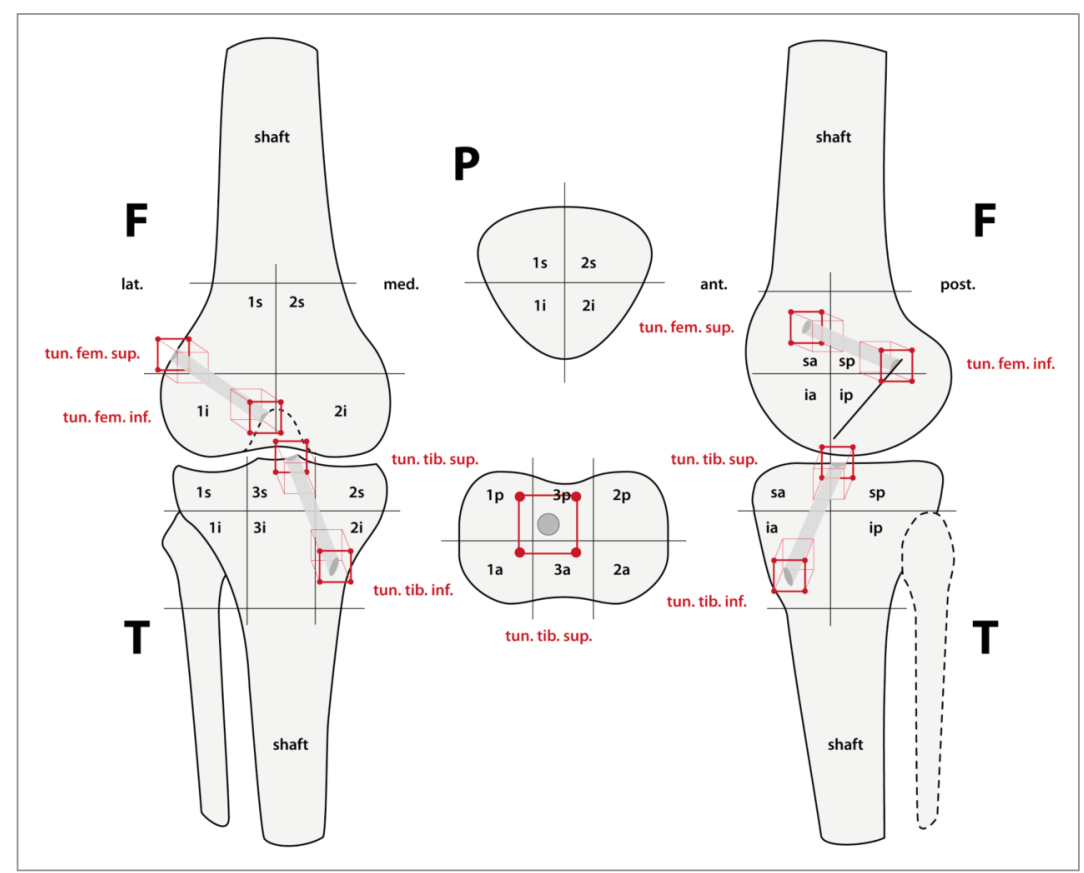

Figure 1. The previously validated localisation scheme for the Tc-99m HDP tracer activity in patients after $\mathrm{ACL}$ reconstruction (1= lateral, 2 = medial, 3 = central). $F$ femur, $T$ tibia, $P$ patella, $a$ anterior, $p$ posterior, $i$ inferior, $s$ superior.

Table 1. Standardised mean and maximum intensity of 99mTc-HDP-BTU in SPECT/CT. Data is presented for each tunnel with regards to graft and fixation type (mean, standard deviation, minimum, maximum, t-test analysis).

\begin{tabular}{|c|c|c|c|c|c|c|c|c|c|c|c|c|c|c|c|c|c|c|c|c|c|c|c|}
\hline & 离 & 竎 & 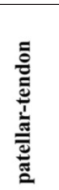 & 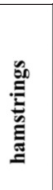 & 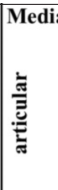 & ian & 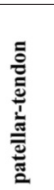 & 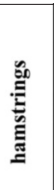 & $\frac{\grave{\omega}}{\bar{E}}$ & 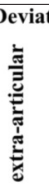 & 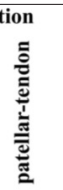 & 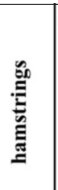 & 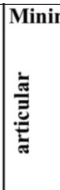 & 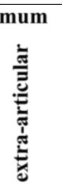 & 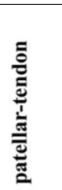 & 岂 & $\frac{\bar{t}}{\bar{E}}$ & mum & 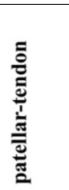 & 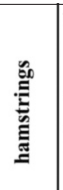 & 氖 & 莺 & fuality of Means \\
\hline $\begin{array}{l}\text { Tunnel femoral } \\
\text { (mean) } \\
\text { Tunnel femoral } \\
\text { (max) } \\
\text { Tunnel tibial } \\
\text { (mean) } \\
\text { Tunnel tibial } \\
\text { (max) }\end{array}$ & $\begin{array}{l}2.03 \\
2.64 \\
2.53 \\
3.77\end{array}$ & $\begin{array}{l}1.92 \\
2.12 \\
2.30 \\
2.84\end{array}$ & $\begin{array}{l}2.01 \\
2.34 \\
2.51 \\
\\
3.57\end{array}$ & $\begin{array}{l}2.23 \\
2.23\end{array}$ & $\begin{array}{l}2.23 \\
2.42 \\
3.39\end{array}$ & $\begin{array}{l}2.24 \\
1.94 \\
2.44\end{array}$ & $\begin{array}{l}2.20 \\
2.10 \\
2.65\end{array}$ & $\begin{array}{l}2.34 \\
1.71\end{array}$ & $\begin{array}{l}1.82 \\
1.79 \\
2.27\end{array}$ & 1.77 & $\begin{array}{l}1.49 \\
1.70 \\
2.28\end{array}$ & $\begin{array}{l}0.77 \\
1.90 \\
1.82\end{array}$ & $\begin{array}{l}0.59 \\
0.47 \\
0.48\end{array}$ & $\begin{array}{l}0.69 \\
0.68 \\
0.73\end{array}$ & $\begin{array}{l}0.59 \\
0.47 \\
0.48\end{array}$ & $\begin{array}{c}0.89 \\
0.68 \\
0.73\end{array}$ & $\begin{array}{l}8.98 \\
9.49 \\
8.88\end{array}$ & $\begin{array}{r}4.30 \\
10.37 \\
9.22\end{array}$ & $\begin{array}{r}8.98 \\
10.37\end{array}$ & $\begin{array}{l}4.19 \\
9.49\end{array}$ & $\begin{array}{l}0.47 \\
1.45 \\
0.48 \\
1.61\end{array}$ & $\begin{array}{l}-0.68 \\
-0.29 \\
-0.57 \\
-1.52\end{array}$ & $\begin{array}{ll}0.637 & 0.499 \\
0.152 & 0.774 \\
0.635 & 0.568 \\
0.113 & 0.136\end{array}$ \\
\hline
\end{tabular}


In vivo bone tunnel remodeling in symptomatic patients after ACL reconstruction: a retrospective comparison of articular and extra-articular fixation

Tunnel enlargement in the femoral tunnel showed significantly higher values of the standardised maximum and mean intensity and distribution of bone tracer uptake in superior femoral regions as well as in the femoral and tibial tunnel and lateral compartment of the tibia. Patients with proximal tibial tunnel enlargement caused significant higher bone tracer uptake in the central area of the tibia as well as in the

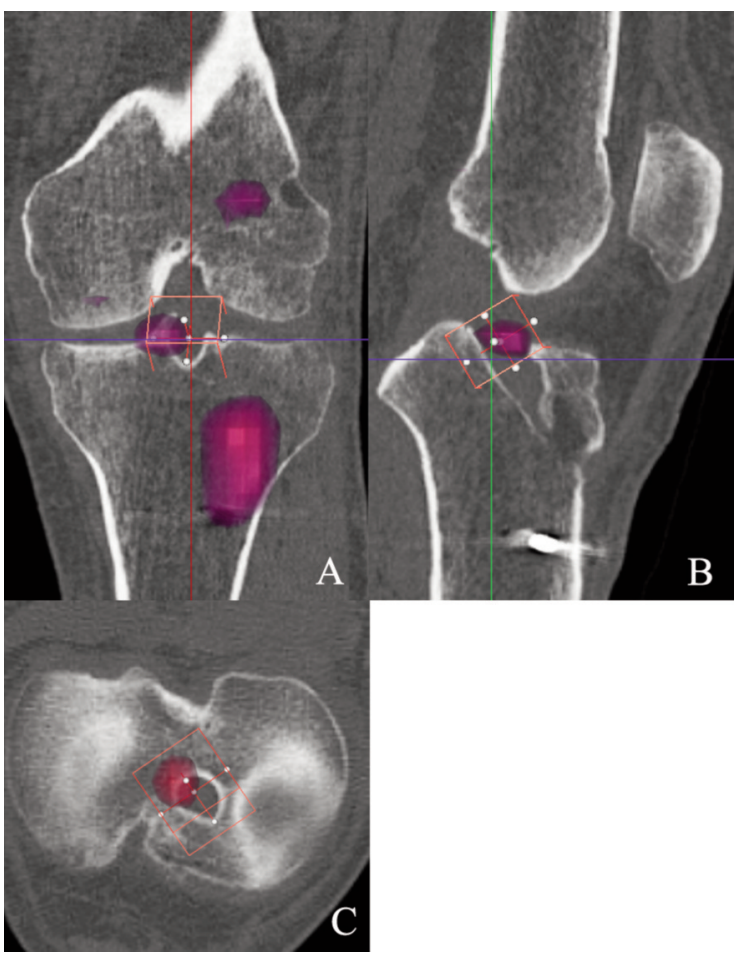

Figure 2. SPECT/CT image of a 48-year-old male patient 9 months after ACL reconstruction using patellar tendon graft with extra-articular fixation showing an significantly increased uptake in the lateral part of the tibia $(A)$ where the graft is fixed. An increased tracer uptake can also be seen in the posterior-central area of the tibia $(B, C)$ indicating advancing metabolic activity. entire tibial tunnel (Tab. 6, Fig. 4). Tibial tunnel length correlated with increased BTU in the femoral tunnel femoral tunnel length with decreased BTU in the medial femoral and tibial compartment (Tab. 7).

\section{Discussion}

\section{Statement of principle findings}

This is the first study investigating in-vivo bone remodeling within the femoral and tibial tunnel in symptomatic patients after ACL reconstruction. Our results confirmed the stated hypothesis, that bone tunnel remodeling is significantly different with regards to the fixation method, graft and tunnel diameter. In clinical practice, the use and value of the SPECT/CT is increasingly recognized in orthopaedic surgery, but the knowledge and evidence about its use is still scarce $^{18,20,21}$. The present study will make an important contribution to improving the understanding of BTU in painful patients after ACL reconstruction and assist in clinical decision-making when choosing fixation technique and graft type.

The most important findings of the study were as follows: Firstly, higher BTU was found for the articular fixation in the femur for the extra-articular fixation and in the tibial tunnel. However, no significant correlation of fixation and graft type with 99mTc-HDP-BTU tracer uptake in SPECT/CT was found for the femoral subgroup. As the sample size is rather small for this study purpose it has to be further investigated in larger scaled studies. In particular the burning question remains if these findings could also be confirmed in patients with normal ACL function (natural history group).

The non-significant findings in the femoral subgroup might be explained by two reasons. One the samples size was not large enough. Two, this is a real finding, which would mean that the femoral fixation is less of a problem for bone remodeling than the tibial one.

Secondly, in the tibial subgroup "patellar tendon graft fixation" showed a significantly higher BTU in the superior-lateral and posterior-central area of the tibia,

Table 2. Standardised mean intensity of 99mTc-HDP-BTU in SPECT/CT for each anatomical femoral region with regards to graft and fixation type (mean, standard deviation, minimum, maximum, t-test analysis).

\begin{tabular}{|c|c|c|c|c|c|c|c|c|c|c|c|c|c|c|c|c|c|c|c|c|c|c|c|c|}
\hline & \multirow[b]{2}{*}{\begin{tabular}{|l} 
Mean \\
$\frac{2}{\frac{0}{3}}$ \\
$\frac{\mathrm{J}}{\mathrm{J}}$ \\
$\frac{\mathrm{t}}{\mathrm{t}}$
\end{tabular}} & \multirow[b]{2}{*}{ 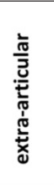 } & \multirow[b]{2}{*}{ 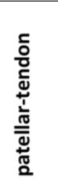 } & \multirow[b]{2}{*}{ 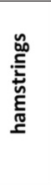 } & \multicolumn{4}{|c|}{ Median } & \multicolumn{4}{|c|}{ Std. Deviation } & \multicolumn{4}{|c|}{ Minimum } & \multicolumn{4}{|c|}{ Maximum } & \multicolumn{4}{|c|}{ t-test for Equality of Means } \\
\hline & & & & & 兽 & 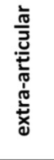 & 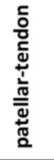 & 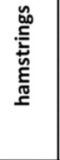 & 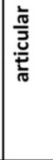 & 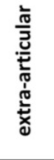 & 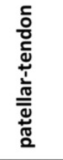 & 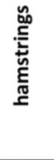 & 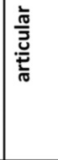 & 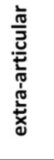 & 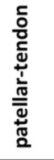 & 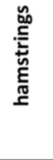 & $\frac{2}{\frac{10}{3}}$ & 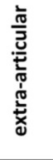 & 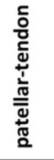 & 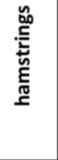 & 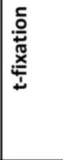 & 壳 & 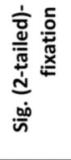 & 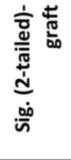 \\
\hline ap.fem.1s_mean & 1.11 & 1.12 & 1.10 & 1.14 & 1.03 & 1.05 & 1.02 & 1.07 & 0.40 & 0.33 & 0.33 & 0.38 & 0.40 & 0.64 & 0.40 & 0.64 & 1.81 & 1.86 & 1.81 & 1.86 & -0.12 & 0.45 & 0.902 & 0.652 \\
\hline ap.fem.2s_mean & 1.01 & 1.00 & 0.99 & 1.03 & 1.01 & 0.95 & 0.92 & 1.01 & 0.38 & 0.31 & 0.35 & 0.32 & 0.32 & 0.51 & 0.32 & 0.61 & 1.94 & 1.59 & 1.94 & 1.57 & 0.04 & 0.40 & 0.968 & 0.691 \\
\hline ap.fem.1i_mean & 1.57 & 1.42 & 1.54 & 1.35 & 1.25 & 1.20 & 1.21 & 1.21 & 0.93 & 0.70 & 0.82 & 0.69 & 0.40 & 0.36 & 0.40 & 0.36 & 4.03 & 3.09 & 4.03 & 3.08 & 0.65 & -0.90 & 0.519 & 0.374 \\
\hline ap.fem.2i_mean & 1.54 & 1.43 & 1.52 & 1.38 & 1.28 & 1.15 & 1.26 & 1.24 & 0.92 & 0.78 & 0.87 & 0.74 & 0.32 & 0.44 & 0.32 & 0.44 & 4.20 & 3.48 & 4.20 & 2.61 & 0.46 & -0.62 & 0.648 & 0.536 \\
\hline sag.fem.sa_mean & 1.05 & 1.03 & 1.01 & 1.08 & 1.03 & 0.96 & 0.96 & 1.05 & 0.37 & 0.29 & 0.29 & 0.35 & 0.39 & 0.62 & 0.39 & 0.62 & 1.79 & 1.79 & 1.79 & 1.79 & 0.24 & 0.83 & 0.812 & 0.412 \\
\hline sag.fem.sp_mean & 0.99 & 1.02 & 1.01 & 1.00 & 1.07 & 0.92 & 0.92 & 1.01 & 0.39 & 0.37 & 0.39 & 0.35 & 0.26 & 0.41 & 0.26 & 0.41 & 1.82 & 1.95 & 1.95 & 1.58 & -0.19 & -0.13 & 0.850 & 0.900 \\
\hline sag.fem.ia_mean & 1.40 & 1.32 & 1.41 & 1.24 & 1.10 & 1.16 & 1.16 & 1.03 & 0.90 & 0.68 & 0.80 & 0.67 & 0.30 & 0.34 & 0.30 & 0.34 & 3.89 & 3.50 & 3.89 & 2.69 & 0.40 & -0.83 & 0.691 & 0.408 \\
\hline sag.fem.ip_mean & 1.62 & 1.49 & 1.59 & 1.44 & 1.43 & 1.35 & 1.35 & 1.36 & 0.81 & 0.75 & 0.78 & 0.74 & 0.37 & 0.45 & 0.37 & 0.45 & 3.44 & 3.60 & 3.60 & 3.03 & 0.57 & -0.72 & 0.574 & 0.475 \\
\hline Fem_mean & 1.14 & 1.09 & 1.13 & 1.07 & 1.08 & 1.02 & 1.07 & 1.05 & 0.55 & 0.43 & 0.49 & 0.44 & 0.31 & 0.46 & 0.31 & 0.46 & 2.55 & 2.10 & 2.55 & 1.95 & 0.38 & -0.46 & 0.703 & 0.648 \\
\hline
\end{tabular}


Table 3. Standardised maximum intensity of 99mTc-HDP-BTU in SPECT/CT for each anatomical femoral region with regards to graft and fixation type (mean, standard deviation, minimum, maximum, t-test analysis).

\begin{tabular}{|c|c|c|c|c|c|c|c|c|c|c|c|c|c|c|c|c|c|c|c|c|c|c|c|c|}
\hline & 离 & 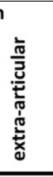 & 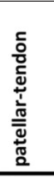 & 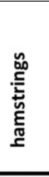 & $\frac{\frac{1}{5}}{\frac{5}{3}}$ & 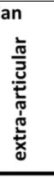 & 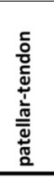 & 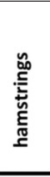 & 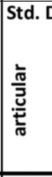 & 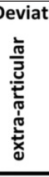 & 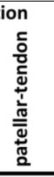 & 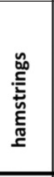 & 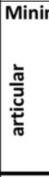 & 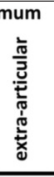 & 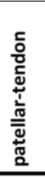 & 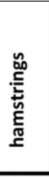 & 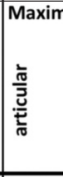 & 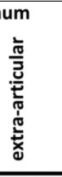 & 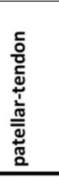 & 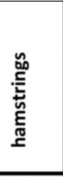 & 党 & 营 & 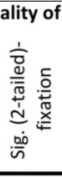 & 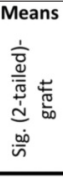 \\
\hline ap.fem.1s_max & 2.51 & 2.44 & 2.56 & 2.30 & 2.24 & 2.35 & 2.31 & 2.38 & 1.08 & 1.08 & 1.26 & 0.65 & 0.86 & 1.09 & 0.86 & 1.17 & 5.19 & 6.84 & 6.84 & 3.43 & 0.23 & -0.89 & 0.819 & 0.379 \\
\hline ap.fem.2s_max & 1.99 & 2.04 & 2.03 & 2.02 & 1.76 & 1.97 & 1.87 & 1.81 & 0.81 & 0.85 & 0.93 & 0.66 & 0.79 & 0.83 & 0.79 & 1.07 & 3.90 & 4.96 & 4.96 & 3.29 & -0.22 & -0.04 & 0.825 & 0.971 \\
\hline ap.fem.1i_max & 3.67 & 2.96 & 3.49 & 2.69 & 3.02 & 2.60 & 2.85 & 2.48 & 2.42 & 1.52 & 2.09 & 1.31 & 0.66 & 0.66 & 0.66 & 0.66 & 10.98 & 6.40 & 10.98 & 5.44 & 1.36 & -1.60 & 0.180 & 0.115 \\
\hline ap.fem.2i_max & 4.20 & 3.50 & 3.92 & 3.40 & 3.21 & 2.61 & 3.01 & 2.75 & 2.96 & 2.44 & 2.62 & 2.61 & 0.59 & 0.77 & 0.59 & 0.77 & 11.38 & 10.42 & 11.38 & 10.42 & 0.94 & -0.73 & 0.353 & 0.469 \\
\hline sag.fem.sa_max & 2.33 & 2.05 & 2.12 & 2.18 & 1.76 & 1.95 & 1.90 & 2.15 & 1.19 & 0.65 & 0.93 & 0.75 & 0.86 & 0.94 & 0.86 & 1.30 & 4.87 & 3.70 & 4.87 & 4.41 & 1.13 & 0.25 & 0.265 & 0.803 \\
\hline sag.fem.sp_max & 2.46 & 2.54 & 2.65 & 2.29 & 2.24 & 2.36 & 2.31 & 2.40 & 1.05 & 1.12 & 1.29 & 0.64 & 0.54 & 1.15 & 0.54 & 1.15 & 5.19 & 6.84 & 6.84 & 3.09 & -0.26 & -1.25 & 0.797 & 0.217 \\
\hline sag.fem.ia_max & 4.19 & 3.59 & 4.05 & 3.36 & 3.24 & 3.01 & 3.32 & 2.97 & 2.84 & 2.22 & 2.58 & 2.15 & 0.77 & 0.79 & 0.77 & 0.79 & 11.38 & 10.27 & 11.38 & 7.79 & 0.86 & -1.05 & 0.393 & 0.300 \\
\hline sag.fem.ip_max & 4.13 & 3.63 & 3.94 & 3.54 & 3.34 & 2.80 & 2.82 & 2.58 & 2.57 & 2.29 & 2.24 & 2.59 & 0.86 & 0.96 & 0.86 & 0.96 & 9.91 & 10.42 & 8.84 & 10.42 & 0.75 & -0.62 & 0.457 & 0.538 \\
\hline Fem_max & 2.83 & 2.53 & 2.75 & 2.42 & 2.69 & 2.20 & 2.42 & 2.18 & 1.51 & 1.11 & 1.33 & 1.08 & 0.66 & 0.97 & 0.66 & 0.97 & 6.86 & 5.32 & 6.86 & 4.87 & 0.85 & -0.98 & 0.396 & 0.332 \\
\hline
\end{tabular}

Table 4. Standardised mean intensity of 99mTc-HDP-BTU in SPECT/CT for each anatomical tibial region with regards to graft and fixation type (mean, standard deviation, minimum, maximum, t-test analysis). Significant correlations and trends in bold, ${ }^{*} p<0.1,{ }^{\star *} p<0.05$.

\begin{tabular}{|c|c|c|c|c|c|c|c|c|c|c|c|c|c|c|c|c|c|c|c|c|c|c|c|c|}
\hline & $\frac{\grave{s}}{\bar{E}}$ & 离 & 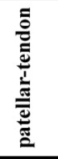 & 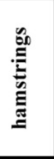 & $\frac{\grave{ \pm}}{\bar{E}}$ & 章 & 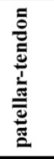 & 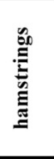 & $\frac{\grave{ \pm}}{\bar{\Xi}}$ & 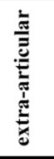 & 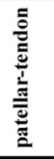 & 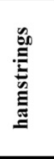 & $\frac{\grave{\Xi}}{\bar{\Xi}}$ & 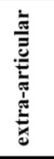 & 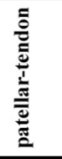 & 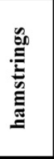 & $\frac{\grave{n}}{\bar{E}}$ & 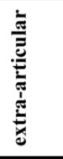 & 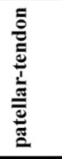 & 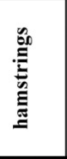 & 竞 & & 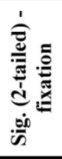 & 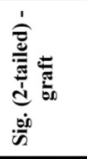 \\
\hline & & & & & & & 2,03 & & & 1,65 & 1,74 & 1,03 & & 0,60 & 1,16 & 0,60 & & & 6,76 & &, 92 & & 0,361 & \\
\hline & & 3 & 3,96 & & & 2,56 & 3,10 & & & 2,18 & 2,35 & 1,63 & 87 & 0,64 & 1,01 &, 64 & & 10,27 & 10,27 & & & $-1,96$ & 0,551 & \\
\hline & & & 3,45 & & 2,06 & 2,63 & 2,31 & 2,37 & & 3,23 & 2,55 & 3,50 & 0,60 & 0,67 & 0,60 & 0,67 & 0,14 & 16,26 & 10,14 & 16,26 & $-0,94$ & & 0,352 & 0,995 \\
\hline & & 57 & 2,62 & 08 & 88 & 2,42 & 2,19 & & & 1,33 & 1,41 & & & 1,02 & 0,51 & 0,98 & 97 & 7,51 & 7,51 & 4,07 & $-1,05$ & $-1,60$ & 0,299 & \\
\hline & & & 2,90 & & & & & & & 1,73 & 1,91 & & & 1,04 & 0,54 & & & 086 & 9,86 & & & & & \\
\hline & & 2,31 & 2,18 & 2,20 & & 1,83 & 1,82 & 1,43 & & 1,37 & 1,25 & 1,56 & & 0,82 & 0,45 & 0,96 & & 5,51 & 5,51 & & & & 0,445 & \\
\hline & 36 & 2,71 & 2,89 & 2,02 & 1,89 & 2,05 & 1,93 & 1,98 & & 1,66 & 1,88 & 0,95 & & 0,60 & 0,93 & 0,60 & 8,04 & 6,57 & 8,04 & & $-0,79$ & $-1,97$ & 0,431 & $0.054^{*}$ \\
\hline & 43 & ,07 & 3,66 & 2,51 & 2,74 & 2,53 & 2,73 & 2,45 & & 1,90 & 2,04 & 1,37 & & 0,57 & 1,16 & 57 & 33 & 9,3 & 9,32 & & 70 & 27 & 0,487 & \\
\hline & 72 & 3,56 & 3,15 & 3,29 & 1,80 & 2,60 & 2,08 & 2,36 & 1,85 & 3,18 & 2,25 & 3,39 & 0,94 & 0,67 & 0,90 & 0,67 & 7,36 & 16,26 & 9,49 & & $-1,15$ & & 0,254 & \\
\hline & & & & & & & & & & 1,43 & & & & 0,42 & 0,63 & & & & & & ,61 & & 0,546 & \\
\hline & 3,57 & 2,87 & 3,50 & 2,61 & 3,01 & 2,38 & 2,54 & 2,51 & 2,05 & 2,12 & 2,28 & 1,68 & 0,76 & 0,63 & 1,15 & 0,63 & 8,32 & 10,27 & 10,27 & 7,79 & 1,24 & $-1,57$ & 0,220 & \\
\hline & & & & & & & & & & 2,24 & & & & & 0,83 & & & & 10,14 & & $-0,05$ & & 0,957 & \\
\hline & 4,13 & 3,85 & 4,23 & 3,55 & 3,21 & 2,83 & 3,83 & 2,85 & 2,67 & 2,90 & 2,60 & 3,08 & 0,77 & 0,63 & 0,77 & 0,63 & 11,38 & 13,57 & 11,38 & 13,57 & 0,37 & $-0,88$ & 0,716 & \\
\hline & & 4,09 & 4,09 & & 2,99 & 3,30 & 3,76 & 2,75 & & 3,08 & 2,24 & 3,41 & & 0,84 & 0,95 & & & & 8,51 & & $-0,38$ & & 0,702 & \\
\hline & & 2,81 & 3,00 & & & 2,28 & 2,53 & & & 1,73 & 1,84 & & & 0,69 & 0,51 & & & 9,86 & 9,86 & & & $-0,42$ & 0,618 & \\
\hline & 2,28 & 2,47 & 2,43 & 2,33 & 1,80 & 2,16 & 1,80 & 2,10 & 1,41 & 1,28 & 1,31 & 1,39 & {$[, 34$} & 1,01 & 0,54 & 1,01 & 6,33 & 5,22 & 5,97 & נכ, & $-0,53$ & $-0,26$ & 0,596 & 0,795 \\
\hline s. & 90 & 01 & 3,15 & 2,65 & 2,46 & 2,50 & 2,66 & 2,39 & 1,57 & 1,66 & 1,64 & 1,54 & 0,92 & 0,83 & 1,12 & 0,83 & 6,95 & 7,98 & 7,98 & 6,78 & $-0,24$ & $-1,14$ & 0,808 & 0,258 \\
\hline
\end{tabular}

meaning the areas of the tibial tunnel near the entrance into the joint. Trends were shown in superiorcentral and lateral region of the tibia for patellar tendon graft. This shows the healing of the bone block at the tunnel entrance site due to more osteoblastic activity. These findings correspond with the results of Mascarenhas et al. ${ }^{22}$ who reported post-operative degenerative changes of grade 1 or higher in the lateral tibio-femoral compartment after ACL reconstruction using bone-patellar tendon-bone autograft compared to hamstring autograft. A higher degree of osteoarthritis was proven to be significantly related to higher bone tracer uptake in the corresponding joint compartments ${ }^{23}$. Furthermore this hypothesis of increased bone remodeling due to graft incorporation and higher osteoblastic activity of the bone-patellar tendon-bone autograft is supported by the study of Ishibashi et al. ${ }^{1}$ as mentioned before.

Thirdly, extra-articular fixation was significantly correlated with higher BTU. The possible cause of these results could include higher tibial bone stress due to intra-tunnel motion of the graft due to extra-articular fixation ${ }^{11}$.

Fourthly, it has been shown that SPECT/CT offers a combined assessment of 3D-CT assessment of the tunnel placement and orientation of the graft together with information on bone remodelling ${ }^{18}$.

Fifthly, this study could prove the correlation of femoral and tibial tunnel diameter with bone tracer uptake as well as tunnel length. The longer the tibial tunnel the more BTU in the femoral tunnel. The longer the femoral tunnel the less BTU in the medial 
In vivo bone tunnel remodeling in symptomatic patients after ACL reconstruction: a retrospective comparison of articular and extra-articular fixation

femoral and tibial compartment. Femoral tunnel enlargement correlated with increased BTU in the femoral and tibial tunnel-tibial tunnel enlargement showed increased BTU only in the tibial tunnel. Presumably tunnel enlargement allows more motion of the graft in the tunnel, and subsequently bone remodeling is taking place.

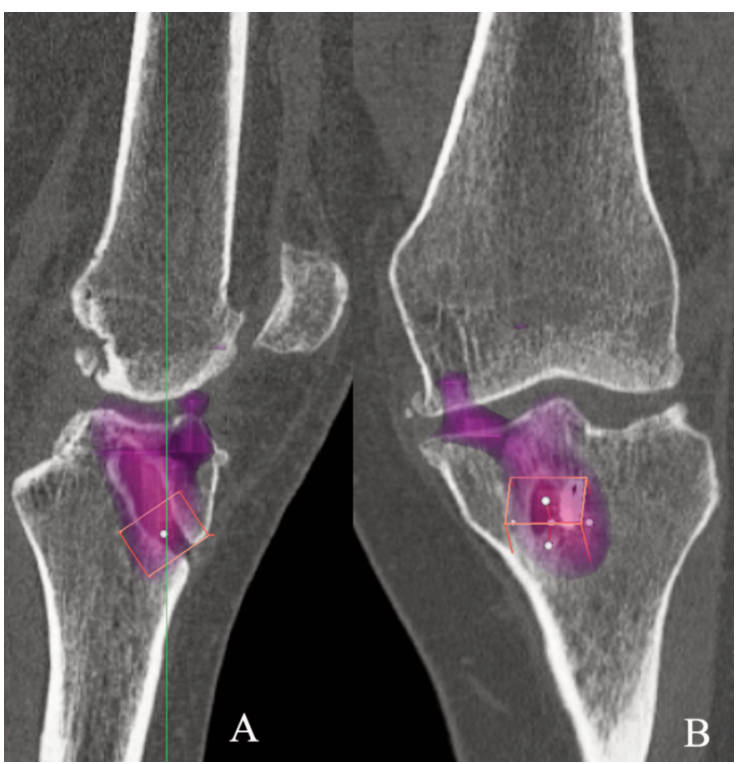

Figure 3. Lateral (A) and anterior-posterior (B) SPECT/CT images of a 31-year-old male patient 8 years after ACL reconstruction using hamstring graft with articular fixation showing bone remodeling along the entire tibial tunnel.
In various recent studies it has been shown that SPECT/CT is able to visualize in vivo joint loading as well as bone remodeling after several surgeries. Hirschmann et al. ${ }^{23}$ reported that SPECT/CT reflects the loading pattern of the knee joint with regards to the mechanical and anatomical alignment. It is also related to the degree of osteoarthritis of the knee. Moreover, the authors found that SPECT/CT could be a useful tool investigating bone tunnel widening, position, remodeling and integration of grafts as well as loading of the medial, lateral and patellofemoral compartment for patients after ACL-reconstruction ${ }^{11,12}$.

However, till this study it was unclear if hamstring autograft or patellar tendon autograft lead to increased BTU and to what extent the distribution of the BTU is influenced by the fixation technique. In this study we have found higher BTU at the tibial entrance site in patients after ACL reconstruction using bone patellar tendon bone autografts representing the healing of the bone block at the tunnel entrance site. Future studies need to differentiate between typical pathologies such as tunnel widening and normal remodeling after $A C L$ reconstruction.

\section{Strengths and weaknesses of the study}

This paper represents the first study to date that analyses the influence of graft type and fixation technique on bone remodeling represented by $99 \mathrm{mTc}$ HDP-BTU in SPECT/CT in various anatomical knee regions. It will further lead to a better understanding of the biology of ACL graft integration and bone re-

Table 5. Standardised maximum intensity of 99mTc-HDP-BTU in SPECT/CT for each anatomical tibial region with regards to graft and fixation type (mean, standard deviation, minimum, maximum, t-test analysis). Significant correlations and trends in bold, ${ }^{*} \mathrm{p}<0.1,{ }^{* *} \mathrm{p}<0.05$.

\begin{tabular}{|c|c|c|c|c|c|c|c|c|c|c|c|c|c|c|c|c|c|c|c|c|c|c|c|c|}
\hline & $\frac{\frac{2}{0}}{\frac{\omega}{3}}$ & 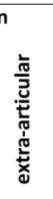 & 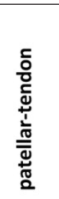 & 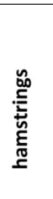 & $\frac{\frac{\hbar}{3}}{\frac{3}{3}}$ & 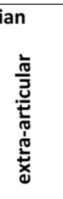 & 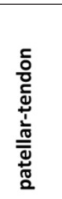 & 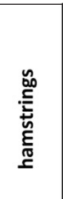 & 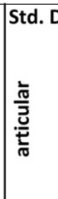 & 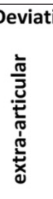 & 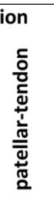 & 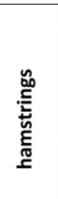 & $\frac{\frac{1}{3}}{\frac{3}{3}}$ & 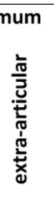 & 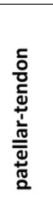 & 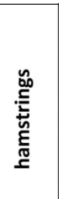 & $\frac{\frac{2}{\sigma 0}}{\frac{d}{2}}$ & 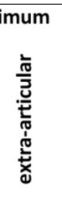 & 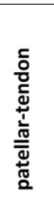 & 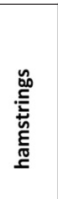 & 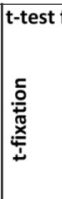 & 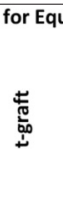 & 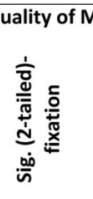 & 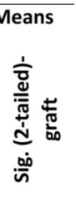 \\
\hline ap.tib.1s_mean & 1,52 & 1,94 & 1,89 & 1,54 & ,36 & 1,63 & 1,55 & 1,42 & 0,77 & 0,99 & 0,97 & 0,79 & 0,57 & 0,48 & 0,68 & 0,48 & 3,60 & 4,41 & 4,41 & 3,58 & $-1,73$ & $-1,38$ & $0.090^{*}$ & 0,172 \\
\hline ap.tib.3s_mean & 1,99 & 2,16 & 2,26 & 1,79 & 1,84 & 1,82 & 1,95 & 1,72 & 1,02 & 1,28 & 1,25 & 0,96 & 0,51 & 0,52 & 0,51 & 0,52 & 4,62 & 7,11 & 7,11 & 4,62 & $-0,51$ & $-1,47$ & 0,610 & 0,147 \\
\hline ap.tib.2s_mean & 1,74 & 2,24 & 2,13 & 1,84 & 1,42 & 1,72 & 1,69 & 1,44 & 1,07 & 1,49 & 1,37 & 1,27 & 0,42 & 0,54 & 0,42 & 0,54 & 4,08 & 6,81 & 6,81 & 5,56 & $-1,42$ & $-0,76$ & 0,162 & 0,452 \\
\hline ap.tib.1i_mean & 1,07 & 1,27 & 1,24 & 1,09 & 1,00 & 1,07 & 1,08 & 0,96 & 0,36 & 0,63 & 0,57 & 0,46 & 0,43 & 0,49 & 0,43 & 0,49 & 1,88 & 3,87 & 3,87 & 2,46 & $-1,40$ & $-0,99$ & 0,167 & 0,329 \\
\hline ap.tib.3i_mean & 1,33 & 1,51 & 1,53 & 1,26 & 1,25 & 1,20 & 1,27 & 0,96 & 0,75 & 0,97 & 0,97 & 0,69 & 0,42 & 0,73 & 0,42 & 0,66 & 3,65 & 5,67 & 5,67 & 3,65 & $-0,73$ & $-1,12$ & 0,467 & 0,269 \\
\hline ap.tib.2i_mean & 0,81 & 0,96 & 0,95 & 0,81 & 0,74 & 0,80 & 0,77 & 0,73 & 0,34 & 0,61 & 0,60 & 0,31 & 0,27 & 0,47 & 0,27 & 0,42 & 1,66 & 3,73 & 3,73 & 1,54 & $-1,13$ & $-0,97$ & 0,263 & 0,339 \\
\hline axial tib.1p_mean & 1,77 & 2,26 & 2,19 & 1,81 & 1,57 & 1,84 & 1,84 & 1,52 & 0,87 & 1,14 & 1,09 & 0,96 & 0,72 & 0,62 & 0,96 & 0,62 & 4,02 & 4,60 & 4,60 & 4,00 & $-1,75$ & $-1,35$ & $0.086^{*}$ & 0,183 \\
\hline axial tib.3p_mean & 1,98 & 2,15 & 2,27 & 1,74 & 1,84 & 1,84 & 1,90 & 1,74 & 0,81 & 1,16 & 1,07 & 0,85 & 0,66 & 0,51 & 1,01 & 0,51 & 3,89 & 5,63 & 5,63 & 3,89 & $-0,62$ & $-1,93$ & 0,535 & $0.059^{*}$ \\
\hline axial tib.2p_mean & 1,73 & 2,50 & 2,26 & 2,03 & 1,54 & 1,95 & 1,77 & 1,60 & 0,79 & 1,70 & 1,38 & 1,51 & 0,58 & 0,66 & 0,94 & 0,58 & 3,58 & 7,48 & 6,35 & 7,48 & $-2,05$ & $-0,58$ & $0.045^{* *}$ & 0,563 \\
\hline axial tib.1a_mean & 1,30 & 1,60 & 1,61 & 1,25 & 1,11 & 1,35 & 1,24 & 1,14 & 0,77 & 0,89 & 0,93 & 0,65 & 0,36 & 0,36 & 0,46 & 0,36 & 3,53 & 4,13 & 4,13 & 3,11 & $-1,35$ & $-1,55$ & 0,184 & 0,127 \\
\hline axial tib.3a_mean & 2,05 & 2,20 & 2,32 & 1,83 & 1,78 & 1,76 & 1,79 & 1,64 & 1,19 & 1,65 & 1,59 & 1,17 & 0,38 & 0,52 & 0,83 & 0,38 & 5,52 & 9,42 & 9,42 & 5,52 & $-0,38$ & $-1,24$ & 0,709 & 0,220 \\
\hline axial tib.2a_mean & 1,77 & 2,06 & 2,05 & 1,74 & 1,27 & 1,62 & 1,54 & 1,35 & 1,29 & 1,55 & 1,57 & 1,19 & 0,42 & 0,41 & 0,64 & 0,41 & 4,99 & 8,27 & 8,27 & 4,98 & $-0,74$ & $-0,77$ & 0,465 & 0,446 \\
\hline sag.tib.sa_mean & 1,68 & 1,97 & 1,98 & 1,62 & 1,47 & 1,62 & 1,60 & 1,47 & 1,10 & 1,29 & 1,32 & 0,99 & 0,42 & 0,40 & 0,42 & 0,40 & 5,03 & 6,99 & 6,99 & 4,20 & $-0,89$ & $-1,07$ & 0,376 & 0,288 \\
\hline sag.tib.sp_mean & 1,65 & 2,10 & 2,02 & 1,71 & 1,60 & 1,88 & 1,85 & 1,80 & 0,69 & 1,07 & 1,01 & 0,81 & 0,58 & 0,57 & 0,58 & 0,57 & 3,38 & 4,67 & 4,67 & 3,46 & $-1,81$ & $-1,22$ & $0.075^{*}$ & 0,229 \\
\hline sag.tib.ia_mean & 1,08 & 1,23 & 1,25 & 1,02 & 0,99 & 1,02 & 1,03 & 0,85 & 0,53 & 0,88 & 0,85 & 0,53 & 0,35 & 0,46 & 0,35 & 0,46 & 2,58 & 5,42 & 5,42 & 2,58 & $-0,77$ & $-1,14$ & 0,442 & 0,260 \\
\hline sag.tib.ip_mean & 0,98 & 1,21 & 1,15 & 1,05 & 0,94 & 1,08 & 1,06 & 0,98 & 0,32 & 0,55 & 0,54 & 0,35 & 0,35 & 0,57 & 0,35 & 0,57 & 1,78 & 2,99 & 2,99 & 1,90 & $-1,78$ & $-0,73$ & $0.080^{*}$ & 0,466 \\
\hline Tib_mean & 1,53 & 1,84 & 1,82 & 1,51 & 1,35 & 1,55 & 1,54 & 1,50 & 0,69 & 0,99 & 0,95 & 0,72 & 0,54 & 0,55 & 0,72 & 0,54 & 3,25 & 5,58 & 5,58 & 3,25 & $\mid-1,31$ & $-1,28$ & 0,197 & 0,207 \\
\hline
\end{tabular}


Table 6. Pearson correlation of standardised maximum and mean intensity of 99mTc-HDP-BTU in SPECT/CT for each anatomical tibial region with regards to tunnel diameter. Significant correlations and trends in bold, * $\mathrm{p}<0.1$, ${ }^{* *} \mathrm{p}<0.05$.

\begin{tabular}{|c|c|c|c|c|c|c|c|c|}
\hline \multirow[b]{2}{*}{ Pearson } & \multicolumn{4}{|l|}{ Mean } & \multicolumn{4}{|c|}{ Maximum } \\
\hline & 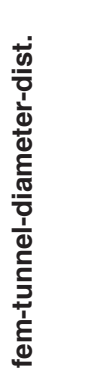 & 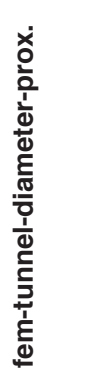 & 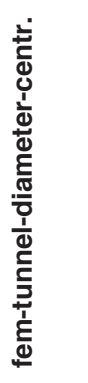 & 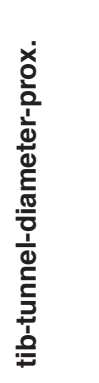 & 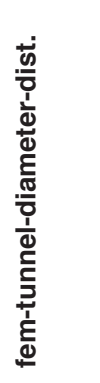 & 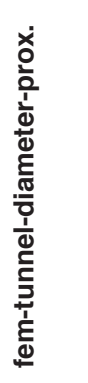 & 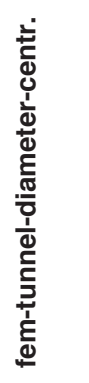 & 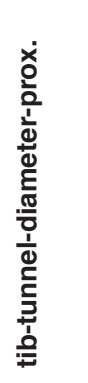 \\
\hline ap.fem.1s & 0.11 & $0.33^{* *}$ & 0.13 & 0.09 & 0.17 & $0.3^{*}$ & 0.19 & 0.01 \\
\hline ap.fem.2s & 0.10 & $0.3^{*}$ & 0.07 & -0.12 & 0.06 & 0.21 & 0.02 & -0.22 \\
\hline ap.fem.1i & 0.08 & 0.18 & 0.07 & 0.09 & -0.06 & 0.03 & -0.04 & 0.12 \\
\hline ap.fem.2i & 0.14 & 0.19 & 0.10 & 0.08 & 0.17 & 0.04 & 0.15 & 0.14 \\
\hline ap.tib.1s & 0.04 & $0.27^{\star}$ & -0.03 & 0.10 & 0.02 & 0.21 & -0.01 & 0.00 \\
\hline ap.tib.3s & 0.20 & 0.09 & 0.09 & 0.13 & 0.21 & 0.01 & 0.14 & 0.13 \\
\hline ap.tib.2s & -0.01 & 0.18 & 0.02 & 0.04 & -0.03 & 0.09 & 0.01 & -0.06 \\
\hline ap.tib.1i & 0.17 & 0.13 & -0.09 & 0.21 & 0.10 & $0.31^{*}$ & -0.01 & 0.19 \\
\hline ap.tib.3i & 0.18 & 0.02 & -0.02 & 0.14 & 0.21 & 0.13 & 0.14 & 0.18 \\
\hline ap.tib.2i & 0.08 & 0.00 & -0.12 & 0.04 & 0.05 & 0.03 & -0.02 & -0.01 \\
\hline axial tib.1p & 0.00 & $0.26^{\star}$ & -0.05 & 0.05 & 0.00 & 0.18 & -0.02 & 0.01 \\
\hline axial tib.3p & 0.19 & 0.07 & 0.06 & 0.03 & 0.23 & -0.01 & 0.14 & 0.15 \\
\hline axial tib.2p & -0.07 & 0.20 & -0.06 & -0.10 & -0.04 & 0.08 & -0.01 & -0.14 \\
\hline axial tib.1a & 0.12 & $0.3^{*}$ & 0.07 & 0.13 & 0.09 & $0.29^{*}$ & 0.15 & 0.07 \\
\hline axial tib.3a & 0.21 & 0.09 & 0.10 & 0.18 & 0.21 & -0.02 & 0.16 & 0.18 \\
\hline axial tib.2a & 0.11 & 0.10 & 0.05 & 0.17 & 0.12 & 0.05 & 0.13 & 0.08 \\
\hline sag.fem.sa & 0.13 & $0.32^{* *}$ & 0.17 & 0.10 & 0.20 & 0.21 & $0.25^{\star}$ & 0.14 \\
\hline sag.fem.sp & 0.07 & $0.29^{\star}$ & 0.02 & -0.11 & 0.16 & $0.26^{*}$ & 0.13 & -0.14 \\
\hline sag.fem.ia & 0.12 & 0.22 & 0.09 & 0.09 & 0.03 & 0.04 & 0.02 & 0.12 \\
\hline sag.fem.ip & 0.11 & 0.18 & 0.09 & 0.05 & 0.09 & 0.04 & 0.08 & 0.12 \\
\hline sag.tib.sa & 0.13 & 0.14 & 0.05 & 0.18 & 0.12 & 0.09 & 0.14 & 0.11 \\
\hline sag.tib.sp & 0.01 & 0.19 & -0.05 & -0.05 & 0.02 & 0.10 & 0.06 & -0.06 \\
\hline sag.tib.ia & 0.21 & 0.07 & 0.01 & 0.20 & 0.20 & 0.15 & 0.17 & 0.23 \\
\hline sag.tib.ip & 0.07 & 0.02 & -0.21 & -0.02 & 0.10 & 0.14 & 0.06 & 0.04 \\
\hline fem.tunnel & 0.18 & 0.20 & 0.22 & 0.22 & $0.3^{*}$ & 0.10 & $0.33^{*}$ & $0.28^{*}$ \\
\hline tib.tunnel & $0.28^{*}$ & -0.03 & 0.13 & $0.28^{\star}$ & $0.33^{* *}$ & -0.03 & $0.26^{*}$ & 0.19 \\
\hline
\end{tabular}
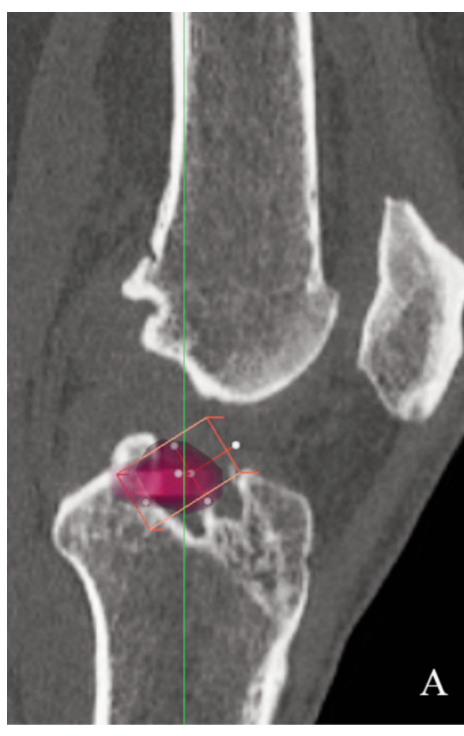

A
Figure 4. Lateral (A) and axial (B) SPECT/CT images of a 46-year-old female patient 6 years after ACL reconstruction using patellar tendon graft with articular fixation showing increased BTU along the tibial tunnel associated with proximal tibial tunnel enlargement. 
In vivo bone tunnel remodeling in symptomatic patients after ACL reconstruction: a retrospective comparison of articular and extra-articular fixation

Table 7. Spearman rho correlation of standardised maximum and mean intensity of 99mTc-HDP-BTU in SPECT/CT for each anatomical tibial region with regards to tunnel length. Significant correlations and trends in bold, ${ }^{*} p<0.1,{ }^{* *} p<0.05$.

\begin{tabular}{cc}
\hline Mean & Maximum \\
\hline
\end{tabular}

Spearman rho

\begin{tabular}{|c|c|c|c|c|}
\hline & 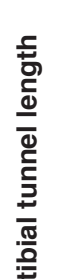 & 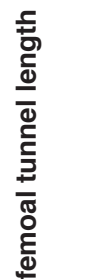 & 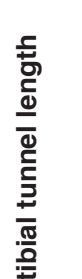 & 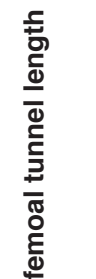 \\
\hline ap.fem.1s & 0.18 & 0.04 & 0.16 & -0.10 \\
\hline ap.fem.2s & 0.03 & 0.03 & -0.01 & 0.01 \\
\hline ap.fem.1i & 0.17 & -0.06 & 0.21 & -0.14 \\
\hline ap.fem.2i & 0.00 & -0.11 & 0.07 & $-0.26^{*}$ \\
\hline ap.tib.1s & 0.10 & -0.03 & 0.12 & 0.01 \\
\hline ap.tib.3s & 0.04 & -0.20 & 0.11 & -0.23 \\
\hline ap.tib.2s & -0.08 & $-0.28^{*}$ & -0.14 & -0.20 \\
\hline ap.tib.1i & 0.13 & 0.07 & 0.11 & 0.07 \\
\hline ap.tib.3i & 0.16 & -0.05 & 0.12 & -0.06 \\
\hline ap.tib.2i & 0.02 & -0.20 & -0.08 & -0.25 \\
\hline axial tib.1p & 0.07 & 0.00 & 0.12 & 0.08 \\
\hline axial tib. $3 p$ & 0.05 & -0.11 & 0.09 & -0.18 \\
\hline axial tib.2p & -0.14 & -0.17 & -0.24 & -0.18 \\
\hline axial tib.1a & 0.05 & -0.12 & 0.02 & -0.14 \\
\hline axial tib.3a & 0.03 & -0.24 & 0.08 & -0.22 \\
\hline axial tib.2a & -0.07 & $-0.29^{*}$ & -0.16 & -0.23 \\
\hline sag.fem.sa & 0.18 & 0.03 & 0.11 & -0.01 \\
\hline sag.fem.sp & 0.06 & 0.00 & 0.07 & -0.03 \\
\hline sag.fem.ia & 0.09 & -0.09 & 0.11 & -0.24 \\
\hline sag.fem.ip & 0.09 & -0.12 & 0.08 & -0.23 \\
\hline sag.tib.sa & 0.03 & -0.23 & 0.08 & -0.28 \\
\hline sag.tib.sp & -0.01 & -0.10 & 0.00 & -0.20 \\
\hline sag.tib.ia & 0.13 & -0.02 & 0.06 & -0.10 \\
\hline sag.tib.ip & 0.08 & -0.02 & 0.00 & -0.08 \\
\hline fem.tunnel & 0.25 & -0.14 & $0.26^{*}$ & -0.08 \\
\hline tib.tunnel & 0.07 & -0.1 & 0.12 & -0.2 \\
\hline
\end{tabular}

modeling within the tunnels after ACL reconstruction. Strengths and weaknesses in relation to other studies: A considerable number of studies have assessed biomechanical differences in graft type and fixation method after ACL reconstruction ${ }^{13-16}$. Most of them used computer or finite element models to calculate changes in stress around the femoral or tibial tunnels. The present study is the only one investigation real in vivo bone turn over within the tunnels after ACL reconstruction.

In a 3D finite element study Yao et al. ${ }^{7}$ found decreased bone stress at lateral and posteromedial regions of the tibial tunnel, whereas it was increased in the subchondral bone at anterior regions of the tunnel. In contrast, in the present study increased $99 \mathrm{mTc}$ HDP-BTU in SPECT/CT was found in lateral and posterior-central areas of the tibia. A finding which appears more biomechanically sound, as higher bone stress is expected when anterior translational force is acting on the graft. More bone stress is represented by higher BTU due to more osteoblastic activity.

Others have shown that bone tendon bone grafts, such as patellar tendon autografts, heal in by osseous incorporation of the bone plug in the bone tunnel (bone-tobone healing by osteoblastic activation), which occurs in a period varying from 3 to 12 weeks ${ }^{1,24}$.

$\mathrm{Au}$ et al. ${ }^{25}$ reported stress alteration occurred around the tibial tunnel wall after the single tunnel creation under the compressive loadings. Abnormal stress distribution near the tunnel may lead to subchondral bone remodeling, which then changes the tunnel morphology.

The following limitations have to be considered when interpreting the study results: retrospective design, moderate sample size, and selection bias caused by only symptomatic postoperative $\mathrm{ACL}$ reconstructions. It could be speculated that the same findings might be found in patients without any problems after ACL reconstruction. In patients with problems after ACL reconstruction the SPECT/CT tracer uptake could be influenced by a variety of factors such as a malposition of the graft, which does not inevitably lead to an altered laxity, but a changed joint kinematics and loading. Meaning of the study: possible mechanisms and implications for clinicians or policymakers.

This study, reported patterns of BTU representing bone remodeling in symptomatic patients after ACL reconstruction.

\section{Acknowledgements}

The Authors would like to thank Mr. Felix Amsler, MSc for statistical analysis of the data.

\section{References}

1. Ishibashi Y, Toh S, Okamura Y, Sasaki T, Kusumi T. Graft incorporation within the tibial bone tunnel after anterior cruciate ligament reconstruction with bone-patellar tendon-bone autograft. The American journal of sports medicine. 2001; 29(4):473-479.

2. Komiyama H, Arai Y, Kajikawa Y, Yoshida A, Morihara T, Terauchi $R$, et al. The fate and role of bone graft-derived cells after autologous tendon and bone transplantation into the bone tunnel. Journal of orthopaedic science: official journal of the Japanese Orthopaedic Association. 2013;18(6):994-1004

3. Lovric V, Kanazawa T, Nakamura Y, Oliver RA, Yu Y, Walsh WR. Effects of Gaps Induced Into the ACL Tendon Graft on Tendon-Bone Healing in a Rodent ACL Reconstruction Model. Muscles, ligaments and tendons journal. 2011;1(3):91-99.

4. Kanazawa T, Soejima T, Noguchi K, Tabuchi K, Noyama M, Nakamura K, et al. Tendon-to-bone healing using autologous bone marrow-derived mesenchymal stem cells in ACL reconstruction without a tibial bone tunnel-A histological study. Muscles, ligaments and tendons journal. 2014;4(2):201-206.

5. Shearer T, Rawson S, Castro SJ, Balint R, Bradley RS, Lowe $\mathrm{T}$, et al. X-ray computed tomography of the anterior cruciate ligament and patellar tendon. Muscles, ligaments and tendons journal. 2014;4(2):238-244. 
6. Lui PP, Ho G, Shum WT, Lee YW, Ho PY, Lo WN, et al. Inferior tendon graft to bone tunnel healing at the tibia compared to that at the femur after anterior cruciate ligament reconstruction. Journal of orthopaedic science: official journal of the Japanese Orthopaedic Association. 2010;15(3):389-401.

7. Yao J, Wen C, Cheung JT, Zhang M, Hu Y, Yan C, et al. Deterioration of stress distribution due to tunnel creation in single-bundle and double-bundle anterior cruciate ligament reconstructions. Annals of biomedical engineering. 2012; 40(7):1554-1567.

8. Neddermann A, Willbold E, Witte F, Hurschler C, Hankemeier $\mathrm{S}$, Stubig $\mathrm{T}$, et al. Tunnel widening after anterior cruciate ligament reconstruction: an experimental study in sheep. The American journal of sports medicine. 2009;37(8):1609-1617.

9. Johnston M, Morse A, Arrington J, Pliner M, Gasser S. Resorption and remodeling of hydroxyapatite-poly-L-lactic acid composite anterior cruciate ligament interference screws. Arthroscopy: the journal of arthroscopic \& related surgery: official publication of the Arthroscopy Association of North America and the International Arthroscopy Association. 2011; 27(12):1671-1678.

10. Weiler A, Peine R, Pashmineh-Azar A, Abel C, Sudkamp NP, Hoffmann RF. Tendon healing in a bone tunnel. Part I: Biomechanical results after biodegradable interference fit fixation in a model of anterior cruciate ligament reconstruction in sheep. Arthroscopy: the journal of arthroscopic \& related surgery: official publication of the Arthroscopy Association of North America and the International Arthroscopy Association. 2002; 18(2):113-123.

11. Hirschmann MT, Mathis $D$, Rasch $\mathrm{H}$, Amsler F, Friederich NF Arnold MP. SPECT/CT tracer uptake is influenced by tunnel orientation and position of the femoral and tibial ACL graft insertion site. International orthopaedics. 2013;37(2):301-309.

12. Hirschmann MT, Mathis D, Afifi FK, Rasch H, Henckel J, Amsler F, et al. Single photon emission computerized tomography and conventional computerized tomography (SPECT/CT) for evaluation of patients after anterior cruciate ligament reconstruction: a novel standardized algorithm combining mechanical and metabolic information. Knee surgery, sports traumatology, arthroscopy: official journal of the ESSKA. 2013; 21(4):965-974.

13. Brand J Jr, Weiler A, Caborn DN, Brown CH Jr, Johnson DL. Graft fixation in cruciate ligament reconstruction. The American journal of sports medicine. 2000;28(5):761-774.

14. Aglietti $P$, Giron F, Buzzi R, Biddau F, Sasso F. Anterior cruciate ligament reconstruction: bone-patellar tendon-bone compared with double semitendinosus and gracilis tendon grafts. A prospective, randomized clinical trial. The Journal of bone and joint surgery American volume. 2004;86-A(10):21432155.

15. Kousa $P$, Jarvinen $T L$, Vihavainen $M$, Kannus $P$, Jarvinen $M$. The fixation strength of six hamstring tendon graft fixation de- vices in anterior cruciate ligament reconstruction. Part II: tibial site. The American journal of sports medicine. 2003; 31(2):182-188.

16. Ibrahim SA, Abdul Ghafar S, Marwan $Y$, Mahgoub AM, Al Misfer A, Farouk H, et al. Intratunnel versus extratunnel autologous hamstring double-bundle graft for anterior cruciate ligament reconstruction: a comparison of 2 femoral fixation procedures. The American journal of sports medicine. 2015; 43(1):161-168.

17. Felmet G. Anatomic Double Bundle single tunnel Foreign Material Free ACL-Reconstruction - a technical note. Muscles, ligaments and tendons journal. 2011;1(4):148-152.

18. Hirschmann MT, Wagner CR, Rasch H, Henckel J. Standardized volumetric 3D-analysis of SPECT/CT imaging in orthopaedics: overcoming the limitations of qualitative 2D analysis. BMC medical imaging. 2012;12:5.

19. Padulo J, Oliva F, Frizziero A, Maffulli N. Muscles, Ligaments and Tendons Journal. Basic principles and recommendations in clinical and field science research. MLTJ. 2013;4:250-252.

20. Hirschmann MT, Konala P, Iranpour F, Kerner A, Rasch H, Friederich NF. Clinical value of SPECT/CT for evaluation of patients with painful knees after total knee arthroplasty-a new dimension of diagnostics? BMC musculoskeletal disorders. 2011;12:36.

21. Hirschmann MT, Adler T, Rasch H, Hugli RW, Friederich NF, Arnold MP. Painful knee joint after ACL reconstruction using biodegradable interference screws- SPECT/CT a valuable diagnostic tool? A case report. Sports medicine, arthroscopy, rehabilitation, therapy \& technology: SMARTT. 2010;2:24.

22. Mascarenhas R, Tranovich MJ, Kropf EJ, Fu FH, Harner CD. Bone-patellar tendon-bone autograft versus hamstring autograft anterior cruciate ligament reconstruction in the young athlete: a retrospective matched analysis with 2-10 year followup. Knee surgery, sports traumatology, arthroscopy: official journal of the ESSKA. 2012;20(8):1520-1527.

23. Hirschmann MT, Schon S, Afifi FK, Amsler F, Rasch H, Friederich NF, et al. Assessment of loading history of compartments in the knee using bone SPECT/CT: a study combining alignment and 99mTc-HDP tracer uptake/distribution patterns. Journal of orthopaedic research: official publication of the Orthopaedic Research Society. 2013;31(2):268-274.

24. Milano G, Mulas PD, Sanna-Passino E, Careddu GM, Ziranu $F$, Fabbriciani $C$. Evaluation of bone plug and soft tissue anterior cruciate ligament graft fixation over time using transverse femoral fixation in a sheep model. Arthroscopy: the journal of arthroscopic \& related surgery: official publication of the Arthroscopy Association of North America and the International Arthroscopy Association. 2005;21(5):532-539.

25. Au AG, Raso VJ, Liggins AB, Otto DD, Amirfazli A. A three-dimensional finite element stress analysis for tunnel placement and buttons in anterior cruciate ligament reconstructions. Journal of biomechanics. 2005;38(4):827-832. 